\title{
Adrenal-sparing surgery for a hormonally active tumour - a single-centre experience
}

\author{
Sadegh Toutounchi, Ryszard Pogorzelski, Tomasz Wołoszko, Ewa Krajewska, Krzysztof Celejewski, \\ Małgorzata Legocka, Wawrzyniec Jakuczun, Zbigniew Gałązka
}

Department of General and Endocrine Surgery, Medical University of Warsaw, Warsaw, Poland

\begin{abstract}
Introduction: Surgeries that spare the adrenal cortex during adrenalectomy have profound justification. Indications for this type of surgery are fairly strict, and more than 30 years of observations continuously verify the results of the procedure.

Material and methods: Of a total of 650 adrenalectomies, 22 (3.4\%) were adrenal cortex-sparing surgeries. There were 16 women and six men in this group. In 10 cases, surgery was performed for pheochromocytoma, eight cases involved Conn's syndrome, and in four cases - paragangliomas located in the para-adrenal region. Secretory activity was identified in all cases.

Results: Laparoscopic partial adrenalectomy was performed in 20 patients. Conversion to open laparotomy was necessary in two cases. In patients after bilateral resection of pheochromocytoma surgery, glucocorticoids were supplemented for six weeks. No significant surgical complications were observed in this group.

Conclusions: Partial adrenalectomy for minor lesions should be a much more commonly utilised treatment method (of choice). Where bilateral adrenalectomy is necessary, a sparing procedure on one side protects patients from the need for hormonal substitution. The remaining part of the adrenal gland undertakes satisfactory secretory function after six weeks at the latest. (Endokrynol Pol 2020; 71 (5): 388-391)

Key words: adrenalectomy; partial adrenalectomy; sparing adrenalectomy
\end{abstract}

\section{Introduction}

Pathology involving both adrenal glands that involves bilateral adrenalectomy is associated with a high risk of complications, such as Addison's syndrome, the incidence of which is estimated at about $10-35 \%$. These patients require hormonal substitution, which is often ineffective, and mortality among this group of patients varies between $3 \%$ and $5 \%$. Partial adrenalectomy, proposed by Irvin et al. in 1983 [1], may be a viable option in some situations. This concerns especially bilateral pheochromocytomas associated with multiple endocrine neoplasia syndromes (MEN, VHL) and single adenomas responsible for excessive production of aldosterone in Conn's syndrome or of cortisol in Cushing's syndrome [2, 3]. It should also be remembered that pheochromocytomas, both associated with genetic syndromes as well as sporadic, may occur asynchronously. It is estimated that $80 \%$ of genetically determined pheochromocytomas are bilateral, while in sporadic cases bilateral manifestation is estimated at about $25 \%$. Also, adenomas of the glomerular layer responsible for overproduction of aldosterone and cortisol occur both synchronously and asynchronously in about $4 \%$ of cases; thus, adrenal cortex-sparing surgeries are fully justified [4]. Currently, the vast majority of surgeries on adrenal glands are performed laparoscopically, but the open method does not eliminate the possibility of leaving a healthy, well-vascularised part of the adrenal cortex [5]. Results of partial adrenalectomy are satisfactory, and the proportion of relapses is acceptable. More than $85 \%$ of patients do not require hormonal substitution [6].

\section{Material and methods}

About 650 adrenalectomies were performed in our centre between 2007 and 2019, including $22(3.4 \%)$ adrenal-sparing operations in patients with hormonally active conditions. This group included 10 patients with pheochromocytoma, eight with Conn's syndrome, and four with paragangliomas located in the immediate vicinity of the adrenal gland. All patients were eligible for laparoscopic adrenalectomy, but in two cases it was necessary to convert to an open procedure.

\section{Pheochromocytoma}

In cases of pheochromocytomas, adrenal-sparing surgery was performed in 10 patients. This group consisted of six women and four men, aged 29 to 61 years, mean 45 years. In six cases, bilateral tumours manifested asynchronously. These patients had undergone one-sided adrenalectomy two to four years earlier. 


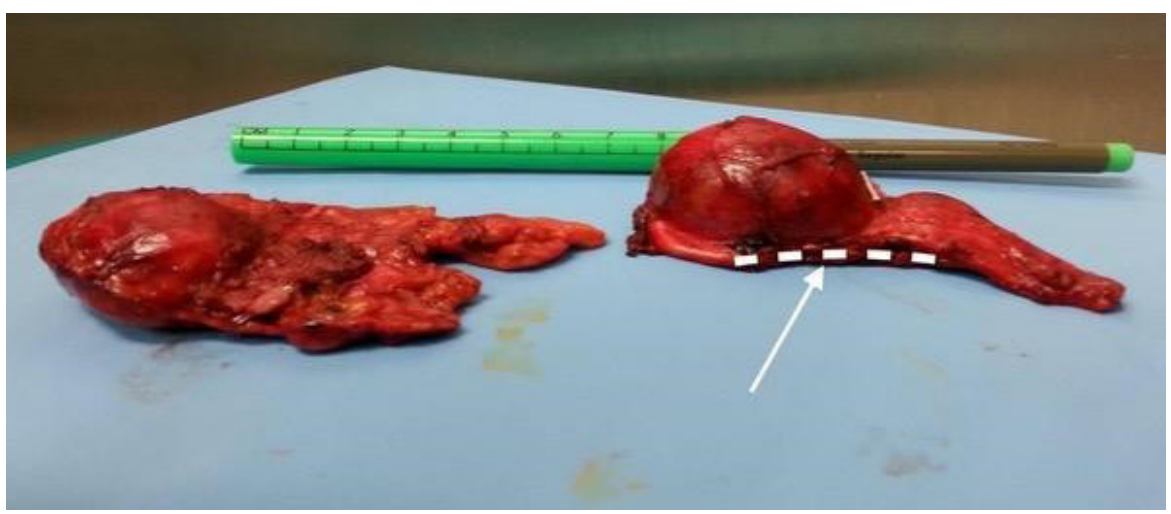

Figure 1. Postoperative specimen from a patient with bilateral pheochromocytoma. On the right side, the entire adrenal gland was removed. On the left side, part of the adrenal cortex was preserved. An arrow indicates the line where the specimen was cut from the remaining part of the adrenal gland

Synchronous tumours of both adrenal glands were diagnosed in the remaining four cases. These patients underwent same-time total adrenalectomy on one side and partial adrenalectomy on the contralateral side (Fig. 1). All patients were referred for laparoscopy, but conversion to open surgery was necessary in a patient with thrombocytopaenia and deranged clotting. Leaving a fragment of the adrenal cortex requires careful circumferential resection of the gland. Afterward, it is usually possible to expose adrenal margins - usually the upper margin with the apex. Intraoperative diagnosis is facilitated by a characteristic orange discoloration of the gland. Since pheochromocytomas are richly vascularised and the adrenal vein is usually large, it was ligated and cut. Arterial branches were cut medially. Leaving the posterior surface of the adrenal gland unresected allows sufficient blood supply to the remaining portion. The tumour is resected from the remaining part of macroscopically healthy adrenal gland using a harmonic knife. During previous surgeries, clips were placed on the remaining adrenal fragment and the tumour was gradually cut off. Glucocorticoid substitution was administered at decreasing doses up to six weeks after surgery. If cortisol levels were normal, substitution was postponed. No perioperative complications were noted in this group of patients. Outpatient follow-up lasting one to five years did not reveal a recurrence of the disease in any case.

\section{Conn's syndrome}

In a similar period, we performed eight adrenal-sparing surgeries in cases of primary hyperaldosteronism. There were six women and two men, aged 37-65years, mean 50.8 years, in this group. Conn's syndrome was confirmed in all cases following comprehensive imaging and laboratory diagnostics. Lateralisation was confirmed by direct catheterisation of adrenal veins with determination of aldosterone levels. In the presented material, in all cases a single adenoma was responsible for the overproduction of aldosterone. The dimensions of adenomas measured in imaging studies and in postoperative histopathological specimens ranged from 15 to $30 \mathrm{~mm}$. Patients were operated on via peritoneal access using four trocars. Adenoma was exposed following careful dissection of the anterior surface of the adrenal gland on either side. Adenomas located eccentrically to the adrenal recess (origin of adrenal vein) can be excised, while leaving arterial blood supply from both middle and inferior adrenal arteries, as well as the venous outflow intact (Fig. 2). This was the case in our patients. We did not notice any perioperative complications in operated patients, and one-year outpatient follow-up did not reveal recurrence of hyperaldosteronism in any case.

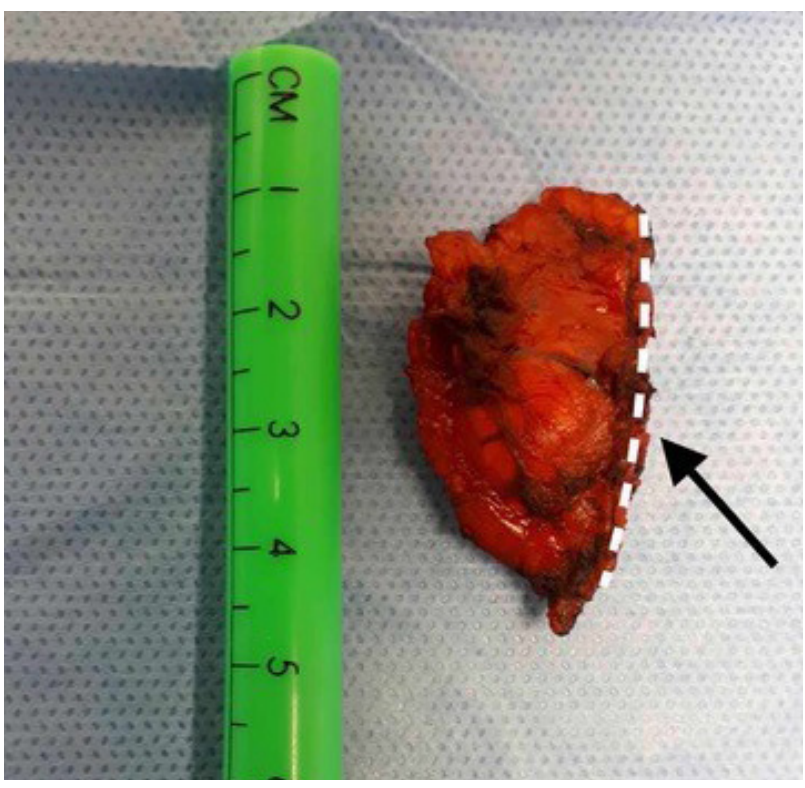

Figure 2. Part of an adrenal gland with a tumour in Conn's syndrome. Dotted line indicates the place where the specimen is resected from a healthy adrenal gland

\section{Adrenal paragangliomas}

Some paragangliomas are located paravertebrally in the immediate proximity of adrenal glands. Imaging diagnostics are often inadequate, and it is not entirely possible to elucidate whether the tumour is located within the adrenal gland or in its immediate neighbourhood [7]. In our group of patients there were four women aged 23 to 64 years, mean 41 years. In all cases, the lesions were located on the right side. Laparoscopy was performed in three patients. During surgery, after dissecting most of the peripheral portion of the lesion, it was found that the largest part of it sits in the lower part of the medial margin. The upper part of the adrenal gland was normal. Therefore, in all cases the upper part of the adrenal gland was preserved, and the tumourous lesion was excised with a harmonic knife and removed. In these cases, the adrenal vein was not cut, leaving anatomical venous drainage from the left portion of the adrenal gland. In one case, open surgery was performed due to the lesion adhering to the lateral surface of the spine and the posterior wall of the inferior vena cava. Intraoperative assessment indicated that it is a paraganglioma located between the vertebra and medial margin of the adrenal gland. After cutting 
elongated adrenal veins the tumour was excised while leaving most of the adrenal gland intact. With the exception of fluctuations in blood pressure during surgery, no other significant perioperative complications were noted. In all cases, histopathology confirmed paraganglioma.

\section{Discussion}

Partial adrenalectomy, or adrenalectomy sparing the cortical layer of adrenal glands, was first introduced more than 30 years ago, before the era of laparoscopy [1]. The first report of partial laparoscopic adrenalectomy dates back to 1997 [8]. Indications for this type of procedure have been established in recent years and, in our opinion, are constantly expanding. The primary indication for sparing adrenalectomy is genetically-determined pheochromocytoma, which occurs bilaterally in about $80 \%$ of cases, followed by Von Hippel-Lindau syndrome and MEN2 syndrome. Sporadic pheochromocytomas can also be bilateral in approximately $25 \%$ of cases, but manifest asynchronously, which also favours sparing surgery [4,9]. Other indications include unilateral adenoma in Conn's or Cushing's syndrome, as well as situations involving bilateral adrenal lesions manifesting synchronously or asynchronously [10]. In our case, a single, usually small adenoma responsible for overproduction of aldosterone is the optimal indication for sparing surgery. In such cases, an almost anatomical resection of the part of adrenal gland with an adenoma can be performed, leaving as much healthy glandular tissue as possible with preserved vascular supply. In autonomous ACTH-independent hypercortisolaemia the situation is a little more complicated because bilateral, nodular cortical hypertrophy may be responsible for this pathology in over $20 \%$ of cases. In our centre we adopted a rule that in ACTH-independent, subclinical forms of hypercortisolaemia, we remove the more enlarged adrenal gland first. We reserve the possibility of sparing adrenalectomy for the opposite adrenal gland. Analysis of our clinical material from patients with this type of pathology has confirmed the legitimacy of such management [11]. In our experience, the greatest problems with sparing adrenalectomy may be associated with pheochromocytoma, especially of cases of larger cortical tumours. More severe bleeding is usually the problem. However, an accurate intraoperative assessment of anatomical relationships in the operating field, performed by an experienced surgeon, usually enables identification of a fragment of adrenal cortex, which is suitable for sparing and producing adrenal steroids at a satisfactory level. Due to the usually disrupted basal part of the adrenal cortex, patients require periodic, postoperative hormonal substitution.

Clinical experience shows that more than $20 \%$ of abdominal paragangliomas occur bilaterally, para- vertebrally, or in the immediate proximity of adrenal glands. Such a location and hormonal hyperactivity often hinder proper diagnosis. The correct diagnosis can be made only during surgery, and then excision of paraganglioma can be performed, while leaving the normal adrenal gland intact [12]. More than 20 years of experience in adrenal laparoscopic surgery, including sparing surgeries and reports on the topic, have enabled the creation of meta-analyses to facilitate critical assessment of partial adrenalectomies. At the moment, we know that in Conn's syndrome and pheochromocytoma, relapse after partial adrenalectomy is observed in about $1-3 \%$ of cases, and more than $90 \%$ of patients do not require long-term hormonal substitution [13]. A slightly higher relapse rate, reaching $10 \%$, is observed after partial adrenalectomy due to pheochromocytoma, especially with a genetic background; hence, this group of patients requires periodic postoperative monitoring [6].

The possibility of an undiagnosed neoplastic process is another problem with partial adrenalectomy. Therefore, patients with small lesions should be referred for this type of surgery, because the likelihood of malignancy correlates with their size. Adrenal tumours with a diameter of less than $6 \mathrm{~cm}$ are accompanied by malignancy in $6 \%$ of cases, while larger tumours - in $25 \%$ of cases. Imaging studies (CT and MR) are often very useful in the diagnostics of adrenal neoplasms. Assessment of some of the tumour's characteristics may bring us closer to the diagnosis $[14,15]$. There were no cases of malignancy diagnosed in postoperative histopathological examination among our patients.

Due to the rapid development of laparoscopic techniques and their widespread availability, some authors believe that sparing surgeries should be the first line of treatment of various adrenal pathologies, both silent as well as hormonally active, especially if the lesions are relatively small, bilateral, and without malignant features [16-18].

\section{References}

1. Irvin GL, Fishman LM, Sher JA. Familial pheochromocytoma. Surgery. 1983; 94(6): 938-940, indexed in Pubmed: 6648808.

2. Walz M, Peitgen K, Hoermann R, et al. Posterior Retroperitoneoscopy as a New Minimally Invasive Approach for Adrenalectomy: Results of 30 Adrenalectomies in 27 Patients. World J Surg. 1996; 20(7): 769-774, doi: 10.1007/s002689900117, indexed in Pubmed: 8678949.

3. Cavallaro G, Polistena A, D'Ermo G, et al. Partial adrenalectomy: when, where, and how? Considerations on technical aspect and indications to surgery. Eur Surg. 2011; 44(3): 150-154, doi: 10.1007/s10353-011-0034-y.

4. Madala A, Daugherty M, Bratslavsky G. Partial Adrenalectomy - Why Should it be Considered? Urology Pract. 2015; 2(6): 359-366, doi: 10.1016/j.urpr.2015.03.006.

5. Miron A, Giulea C, Nădrăgea M, et al. -. Laparoscopic Partial Adrenalectomy. Chirurgia (Bucur). 2017; 112(1): 77-81, doi: 10.21614/chirurgia.112.1.77, indexed in Pubmed: 28266298.

6. Nagaraja V, Eslick GD, Edirimanne S. Recurrence and functional outcomes of partial adrenalectomy: a systematic review and meta-analysis. Int J Surg. 2015; 16(Pt A): 7-13, doi: 10.1016/j.ijsu.2015.01.015, indexed in Pubmed: 25681039. 
7. Toutounchi S, Legocka ME, Pogorzelski R, et al. Surgical treatmen of abdominal paragangliomas. Endokrynol Pol. 2019; 70(6): 469-472, doi: 10.5603/EP.a2019.0038, indexed in Pubmed: 31529458

8. Janetschek G, Lhotta K, Gasser R, et al. Adrenal-sparing laparoscopic surgery for aldosterone-producing adenoma. J Endourol. 1997; 11(2) 145-148, doi: 10.1089/end.1997.11.145, indexed in Pubmed: 9107590.

9. Volkin D, Yerram N, Ahmed F, et al. Partial adrenalectomy minimizes the need for long-term hormone replacement in pediatric patients with pheochromocytoma and von Hippel-Lindau syndrome. J Ped Surg. 2012; 47(11): 2077-2082, doi: 10.1016/j.jpedsurg.2012.07.003, indexed in Pubmed: 23164001.

10. Otto M, Dzwonkowski J. Adrenal-preserving surgery of adrenal tumours. Endokrynol Pol. 2015; 66(1): 80-96, doi: 10.5603/EP.2015.0012, indexed in Pubmed: 25754286.

11. Pogorzelski R, Toutounchi S, Ambroziak U, et al. Effectiveness of unilateral laparoscopic adrenalectomy in ACTH-independent hypercortisolaemia and subclinical Cushing's syndrome - a retrospective study on a large cohort. Endokrynol Pol. 2018; 69(4): 411-415, doi: 10.5603/EP.a2018.0039, indexed in Pubmed: 29952409.

12. Soczomski P, Jurecka-Lubieniecka B, Rogozik N, et al. Multiple endocrine neoplasia type 1 in Poland: a two-centre experience. Endokrynol
Pol. 2019; 70(5): 385-391, doi: 10.5603/EPa2019.0031, indexed in Pubmed: 31274185

13. Kaye DR, Storey BB, Pacak K, et al. Partial adrenalectomy: underused first line therapy for small adrenal tumors. J Urol. 2010; 184(1): 18-25, doi: 10.1016/j.juro.2010.03.052, indexed in Pubmed: 20546805.

14. Silvinato A, Bernardo WM, Branco AW. Total and partial laparoscopic adrenalectomy. Rev Assoc Med Bras (1992). 2019; 65(5): 578-585, doi: 1 0.1590/1806-9282.65.5.578, indexed in Pubmed: 31166429.

15. Jeschke K, Janetschek G, Peschel R, et al. Laparoscopic partial adrenalectomy in patients with aldosterone-producing adenomas: indications, technique, and results. Urology. 2003; 61(1): 69-72; discussion 72, doi: 10.1016/s0090-4295(02)02240-9, indexed in Pubmed: 12559268.

16. Diner EK, Franks ME, Behari A, et al. Partial adrenalectomy: the National Cancer Institute experience. Urology. 2005; 66(1): 19-23, doi: 10.1016/j. urology.2005.01.009, indexed in Pubmed: 15961144.

17. Sateya S, Richardsa N, Brodya F. Bilateral Laparoscopic Partial Adrenalectomy: A Case Presentation and Review of the Literature. J Med Cases. 2014; 5: 212-216, doi: 10.14740/jmc1708w.

18. Colleselli D, Janetschek G. Current trends in partial adrenalectomy. Curr Opin Urol. 2015; 25(2): 89-94, doi: 10.1097/MOU.0000000000000147, indexed in Pubmed: 25581540. 